\title{
On Network Topology Augmentation for Global Connectivity under Regional Failures
}

\author{
János Tapolcai*, Zsombor L. Hajdú*, Alija Pašić*, Pin-Han Ho ${ }^{\dagger}$, Lajos Rónyai ${ }^{\ddagger}$ \\ ${ }^{*}$ MTA-BME Future Internet Research Group, Faculty of Electrical Engineering and Informatics (VIK), \\ Budapest University of Technology and Economics (BME), \{tapolcai,hajdu,pasic\}@ tmit.bme.hu \\ $\dagger$ Dept. of Electrical and Computer Engineering, University of Waterloo, Canada, p4ho@uwaterloo.ca \\ ‡ Institute for Computer Science and Control, Eötvös Loránd Research Network (ELKH SZTAKI) and BME, ronyai@sztaki.hu
}

\begin{abstract}
Several recent studies shed light on the vulnerability of networks against regional failures, which are failures of multiple nodes and links in a physical region due to a natural disaster. The paper defines a novel design framework, called Geometric Network Augmentation (GNA), which determines a set of node pairs and the new cable routes to be deployed between each of them to make the network always remain connected when a regional failure of a given size occurs. With the proposed GNA design framework, we provide mathematical analysis and efficient heuristic algorithms that are built on the latest computational geometry tools and combinatorial optimization techniques. Through extensive simulation we show that augmentation with just a small number of new cable routes will achieve the desired resilience against all the considered regional failures.
\end{abstract}

\section{INTRODUCTION}

The Internet backbone's physical infrastructure consists of thousands of kilometers of undersea, aerial, and buried fiberoptic cables. Several natural disasters, such as earthquakes, hurricanes, and tsunamis, may destroy a number of nodes and links located in possibly a few hundred kilometers wide geographic area [1]-[9], leading to a regional failure.

Since the area of a regional failure is relatively small compared to the size of the backbone, the question naturally comes up: how to ensure the residual network connectivity in the presence of such a failure event? Several techniques are reported to enhance a network topology so as to survive through a regional failure, which can be summarized into the following three classes.

(1) Purchase existing dark, shared fiber assets [10], [11], which is the most common method.

(2) Shield existing fibers [12], [13], at the expense of significantly increased network cost.

(3) Install new fiber cables [14], [15], which is effective only if the new cables are properly placed.

This study follows the strategy of (3), where the problem is to determine which node pairs are allocated with new cable routes (or links), and once we have these node pairs, how the links are geographically placed/routed to achieve the required resilience to any possible regional failure of a given size. Here, the required network resilience is said to be achieved if there are no isolated components in the presence of any regional failure.
Regional failures can be described with the help of Shared Risk Link Groups (SRLGs), and many of the previously reported studies were investigating the protection of SRLGs [16]-[19], mostly in the aspects of failure modeling, network planning, and survivable routing [13], [20]-[24].

Several insightful works were reported on how to model a regional failure event [1]-[9], [25], [26], most of which assume a given shape of an enclosing area for the considered disasters, such as a circular disk. These studies are essential for creating real-like failure scenarios; however, they do not contribute directly to enhancing the survivability of the networks.

In [27]-[29], the possibility of utilizing geo-diverse routing is discussed for increasing network survivability of regional failures, where the spatial separation between disjoint paths can be ensured. This study also assumes that any network equipment covered by a circular disk centered at the epicenter of a given radius, say $50 \mathrm{~km}$, could fail. Note that these studies can ensure service continuity in the presence of regional failures if the survived part of the network remains connected upon a failure, which may not always be the case. This motivates another design dimension via network planning that aims to create a regional failure survivable network [12], [30][32].

The impacts of natural disasters on terrestrial and submarine cables have been assessed in [1], [5], [33]-[35] and [36], [37], respectively. In [14], a disaster-aware submarine cable deployment algorithm was devised by exploring a greenfield network planning approach, which avoids the network infrastructure to be deployed in the disaster areas. In [15], a cost-effective approach was presented for planning submarine cable routes (paths) with minimized overall life-cycle cost of the submarine cables. By assuming irregular shapes of the cable routes, an Integer Linear Program (ILP) was formulated for physical path selection, whose optimality is nonetheless affected by some additional constraints. Note that the study did not consider to plan several paths simultaneously.

In [38], the concept of multiple region-based connectivity was introduced as a metric for massive yet localized faults, which again leveraged the greenfield fault-tolerant network design principle. In [39] as a strategy to strengthen the resilience of optical networks against disasters, the concept of emergency optical networks and hierarchical addressing was investigated. 
In [40], stochastic models and risk-minimizing node relocation schemes were proposed by manipulating where each node should be moved. In [15], the problem of how to lay down a single cable (or how to find the path) to connect two points across an Earthquake Fault Line was considered. The paper formulates the problem as multi-objective optimization of cable cost and failing probability of the cable, and further provides meaningful insights on the possible cable shape alternatives.

In our paper, we take a completely different angle from all the previous research in the sense that all the nodes are static in the graph (i.e., no reallocation is considered), while the network is augmented by adding multiple cable routes/links to create regional failure resilient networks. The main contributions of this paper are listed as follows:

- We introduce a novel framework of regional failure protection via link augmentation. Due to the high complexity of the formulated problem, an efficient heuristic algorithm is provided where the original problem is divided into a master problem and a sub-problem.

- The sub-problem tackles how to add links between given end nodes concerning each possible regional failure. Here we solve the problem via the latest computational geometry tools that show great effectiveness. We prove several vital properties of the optimal cable routes.

- We introduce three algorithms to solve the master problem, that aim to select the node pairs to connect by new edges. For each edge it also computes the cable routes, end ensures that every possible regional failure is protected by a new edge.

- We provide extensive simulation results to assess the performance of the proposed algorithms in terms of total cable length and required runtime, followed by detailed discussions and insights on the proposed problem and design framework. We conclude that our approaches can find near-optimal augmentation with decent computation time for numerous real-world network topologies.

The rest of the paper is organized as follows. Section II presents the system model. Section III discusses the computational geometry sub-problem, called Shortest Detours Problem (SDP), which serves as the building block of the proposed design framework. In Section IV, we present the proposed algorithmic framework for the Geometric Network Augmentation (GNA) Problem. In Section V we present the experimental results. Section VI concludes our paper.

\section{MODEL And Assumptions}

The network is modeled as an undirected connected geometric graph $\mathcal{G}=(\mathcal{V}, \mathcal{E})$ with $n=|\mathcal{V}|$ nodes and $m=|\mathcal{E}|$ edges, $m \geq n-1$. The nodes of the graph are embedded as points in the Euclidean plane, and each edge is considered to be a line segment. Note that it is straightforward to extend this study to the scenario where the routes of physical fibers are modeled as polygonal chains specified by sequences of corner points.
The paper utilizes the disk failure model with fixed radius, where a regional failure occurs at a point known as the epicenter that corresponds to an area in a shape of circular disk $c$ of radius $r$. All network elements intersecting with the area are affected, and all other network elements are unaffected.

Definition 1. A circular disk failure $c$ hits an edge $e$ if $e$ intersects the interior of disk $c$. Similarly node $v$ is hit by failure $c$ if it is in the interior of $c$. Let $\mathcal{E}_{c}$ (and $\mathcal{V}_{c}$ ) denote the set of edges (and nodes) hit by disk $c$.

Definition 2. A network survives a circular disk failure $c$ if the graph $\mathcal{G}_{c}=\left(\mathcal{V} \backslash \mathcal{V}_{c}, \mathcal{E} \backslash \mathcal{E}_{c}\right)$ is connected. Otherwise, if $\mathcal{G}_{c}$ is not connected, then the center of $c$ is called a danger point.

The applicability of the above definitions is straightforward if the affected regions are of circular shape. For many disasters, the damage is mainly determined by the physical distance to the epicenter, e.g., attacks via mass destruction weapons. However, some natural disasters cause a damaged area of a specific shape; e.g., tsunamis damage areas near the sea shore, earthquakes are likely to happen in seismic zones, and flooding can happen along a riverside [41]. Nevertheless, we argue that our definitions are relevant in these scenarios as well, where the nodes/links may not be affected by failure even if they fall within the disk of the failure. The intuition is that the nodes in the disk are not protectable with a reasonable cost from the corresponding failure event and thus their connection to the network cannot be guaranteed. Instead of dealing with the problem of maintaining service continuity in the catastrophic region, our goal of this study is to guarantee the Internet connectivity for those nodes far enough from the center of the disaster.

To reach our goal, we take the strategy of (3) by installing new fiber-optic cables. For easier understanding, we assume that the cost of adding a new cable is proportional to its physical length, although in reality the costs greatly depend on the terrain and other factors.

Section III-A will discuss how to modify the proposed design framework for a general cost function of installing cables. The proposed GNA framework aims to find the minimum cost network topology augmentation to survive a single circular disk failure of radius $r$ at any location. The problem of GNA is given as follows.

\section{Geometric Network Augmentation (GNA) Problem}

Input : A network represented by an undirected geometric graph $\mathcal{G}=(\mathcal{V}, \mathcal{E})$, where the nodes are embedded as points, and the edges as line segments in the Euclidean plane, and maximum radius $r$ of a possible regional failure.

Output: Add curves as new edges to $\mathcal{E}$, such that the network survives any circular disk failure of radius $r$ (see Def. 2), and the total length of the curves is minimal. 


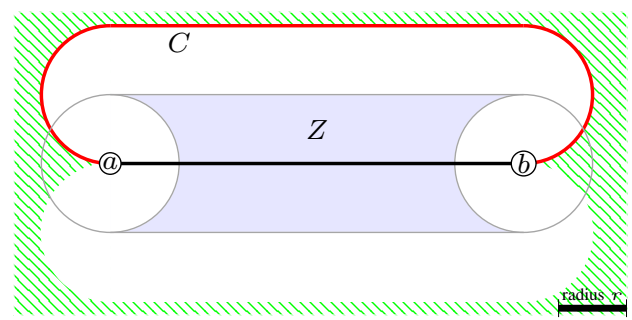

(a) Long edge, $|a b| \geq 4 r$

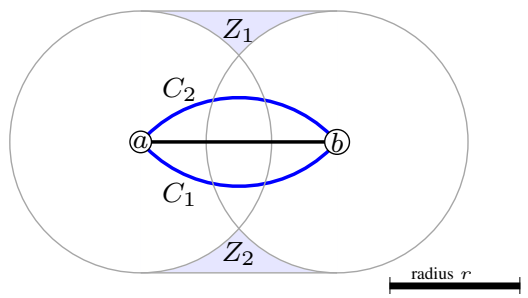

(b) Very short edge, $|a b|<2 r$

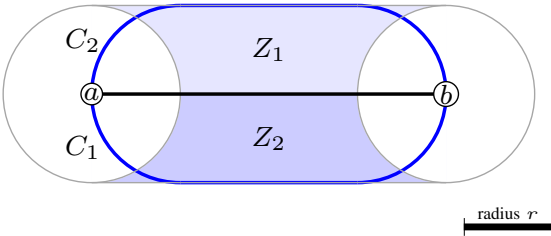

(c) Short edge, $2 r \leq|a b| \leq 4 r$

Fig. 1. A network of two nodes and a link. The danger zones are colored blue, where the radius $r$ of the circular disk is drawn as a line segment in the bottom of the figure. These are the optimal solutions. For brevity, we omit the proof here.

\section{A. Example Network}

The proposed GNA problem is demonstrated via a simple example, as shown in Fig. 1a. According to Def. 2 the network is survivable to any circular disk failure with its center closer than $r$ to any of the nodes. It is because the network would have one active node after the failure. The network also survives if the center of the disk is farther than $r$ to the link. The rest of the points on the plane are the danger points, by which the network is separated into two disjoint nodes and does not survive the failure.

Definition 3. A danger zone is a connected set of danger points in the plane.

The network in Fig. 1a has a single danger zone colored in blue. To make the network survive any disk failure of radius $r$, we need to add an edge of a curve with a distance of at least $r$ to the danger zone. The complement of the $r$ distance neighborhood of the danger zone, colored in green, is where the new edge can traverse through. The curve in red shown in the figure is a feasible solution to the simple example.

The above example assumes that the link connecting the two nodes is much longer than $r$. If the link is shorter than $4 r$, a more economical solution is to add two new edges, see Fig. $1 \mathrm{~b}$ and 1c. For $|a b|<2 r$, the set of danger points form two connected areas; i.e., we have two danger zones. Similar to Fig. 1a, each new edge is going to be an arc with distance $r$ from one of the danger zones.

It is interesting to note that adding two new edges is cheaper than a single edge (like Fig. 1a) for $2 r \leq|a b| \leq 4 r$, where we have a single danger zone $Z$, but it is more economical to cut it into two areas, denoted by $Z_{1}$ and $Z_{2}$, along line segment $a b$, the new edge $C_{1}$ (and $C_{2}$ ) is a curve with $r$ distance from $Z_{1}$ $\left(Z_{2}\right.$, resp.) the halved danger zone. Since $Z_{1} \cup Z_{2} \equiv Z$, every danger point is protected by either curve $C_{1}$ or $C_{2}$. Note that if the length of the edge is $4 r$, both solutions, i.e., the single edge (Fig. 1a) and the two edge (Fig. 1c) solutions, have an equal cost.

\section{Algorithms to Compute Optimal Cable Routes}

In this section, we define the Shortest Detours Problem (SDP) for a single pair of nodes, which serves as the building block in solving the GNA problem. We will first present the single curve and multi-curve cases, followed by a heuristic algorithm for the multi-curve scenario.

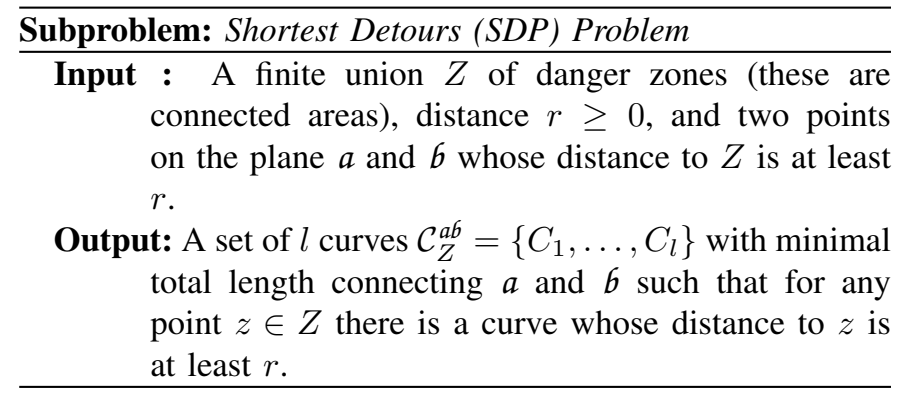

\section{A. Single Curve SDP Solution}

If the optimal solution is a single curve (i.e., $l=1$ ), after offsetting (inflating) $Z$ by radius $r$, the problem can be solved with the geometric Dijkstra algorithm: computing the shortest path between two points in the presence of obstacles [42]-[44]. The corresponding computational geometry problem received particular attention in the last decades for various emerging application scenarios, such as robot motion planning in a warehouse setting. Constant radius offsetting for curves and surfaces is also a widely studied geometric operation due to its immediate application in manufacturing, which involves the use of computers to control machine tools such as drills and lathes. In general, offsetting is one of the most difficult geometric operations in the sense of implementation [45]. In this study, nonetheless, the SDP is a special case of offsetting where the areas are bounded by line segments and circular arcs only, leading to a problem that is easy to solve [46].

Claim 4. The optimal solution of the SDP with $l=1$ and uniform cost on danger zone $Z$ can be found in polynomial time. The obtained curve $C$ is defined as the shortest $r$-detour of $Z$.

Proof: Offsetting areas bounded by line segments and circular arcs can be done in polynomial time [46]. The worst-case complexity of geometric Dijkstra algorithm is $O\left(n^{\prime} \log n^{\prime}\right)$, where $n^{\prime}$ is the total number of vertices in the obstacle polygons [44]. In our case, $n^{\prime}$ is bounded by a polynomial function of the number of links $m$.

Note that there are efficient open-source implementations for offsetting and the geometric Dijkstra algorithm. Further, 


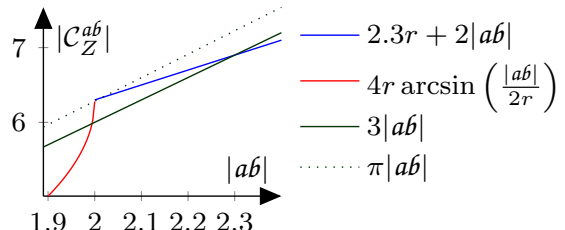

Fig. 2. The upper bounds of Claim 5 for $r=1$. It is easy to verify that the bounds are at most $\pi|a b|$, thus there cannot be more than 3 curves in the optimal solution. Note that the bound is larger than $3|a b|$ only if $1.99 r<|a b|<2.3 r$.

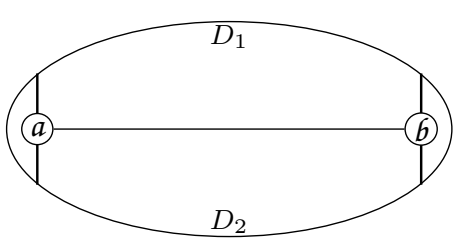

Fig. 3. The illustration of the proof of Theorem 1. The three curves $C_{1}, C_{2}$, $C_{3}$, must entirely be inside the ellipse $\mathcal{E}$ with foci $a$ and $b$ and major axis of length 1.16 .

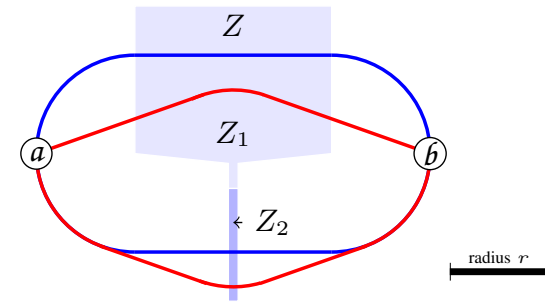

Fig. 4. An example of $Z$ when the division of $Z$ through line $a b$ is not optimal. The blue curves correspond to $Z$ divided by line $a b$, and the red curves are the optimal solution, where $Z$ is divided into $Z_{1}$ and $Z_{2}$ as shown. algorithms to handle cable installation costs are available on GPU [47]. It can consider "traverse speed" when constructing a path from a source point to every point on the plane. In our case, the traverse speed would be the cost of installing a new fiber on each location. This local cost depends on the existing infrastructure, terrain, and other factors.

\section{B. Multi-Curve SDP Solution $(l \geq 2)$ for Uniform Cost}

The examples of Fig. $1 \mathrm{~b}$ and $1 \mathrm{c}$ motivate us to deal with the case when $l=2$ or even larger $l$. For $l=2$ we divide $Z$ into two disjoint parts $Z_{1}$ and $Z_{2}$, to obtain $C_{1}$, the shortest $r$-detour curve for $Z_{1}$, and $C_{2}$, the shortest $r$-detour curve for $Z_{2}$. Note that $C_{1}$ and $C_{2}$ protects every point in $Z_{1}$ and $Z_{2}$, respectively, and only one out of the two curves operates in case of a disaster with an epicenter in $Z$, since $Z \equiv Z_{1} \cup Z_{2}$. In the following paragraphs, we give an upper bound on the length of the curves based on the blue solution in Fig. 1c.

Claim 5. Let $\mathcal{C}_{Z}^{a b}$ be optimal curves for SDP for uniform cost. Then the total length of the curves is at most (see also Fig. 2)

$$
\begin{array}{ll}
\left|\mathcal{C}_{Z}^{a b}\right|<2.3 r+2|a b|, \quad \text { if }|a b| \geq 2 r \text { and } \\
\left|\mathcal{C}_{Z}^{a b}\right| \leq 4 r \arcsin \left(\frac{|a b|}{2 r}\right), & \text { otherwise, }
\end{array}
$$

where $|a b|$ denotes the distance between points $a$ and 6 .

Proof: In the proof we will provide a feasible solution and determine its cost. For the first inequality we take the blue solution in Fig. 1c, where both curves start and terminate with a quarter circle of radius $r$ followed by a line segment parallel with the line through points $a$ and $b$. The length of each quarter circle is $\frac{1}{2} r \pi$, while the line segment is $|a b|-2 r$ long. One can verify that this is always a valid solution because, $a$ and $b$ are outside of $Z$ with distance at least $r$. In the solution there are two curves, denoted by $C_{1}$ and $C_{2}$, and their length is

$\left|\mathcal{C}_{Z}^{a b}\right| \leq\left|C_{1}\right|+\left|C_{2}\right|=4 \cdot \frac{1}{2} r \pi+2 \cdot(|a b|-2 r)<2.3 r+2|a b|$.

For the second inequality, we take the solution in Fig. 1b, where the two paths are two arcs of a circle with radius $r$. Let $\alpha$ denote the half of the angle of the arc, where we have $\sin \alpha=\frac{|a b|}{2 r}$. Thus $\alpha=\arcsin \left(\frac{|a b|}{2 r}\right)$, and the length of each $\operatorname{arc}$ is $2 r \arcsin \left(\frac{|a b|}{2 r}\right)$.
Theorem 1. The number of curves in the optimal solution is $l=1$ or 2 for uniform cost.

Proof: Suppose for contradiction that the optimal solution for an instance of SDP consists of three curves $C_{1}, C_{2}, C_{3}$. Without loss of generality, we assume that $a, b$ are on a horizontal line and $|a b|=1$. Then Claim 5 implies that $1.99 r \leq|a b| \leq 2.3 \cdot r$ (the first inequality follows from the fact that the function $3 x-4 \arcsin (x / 2)$ stays positive on the interval $(0,1.99])$, hence $0.51>r>0.4$. Due to the fact that the total length of the solution is at most $\pi|a b|$, we obtain that $1 \leq\left|C_{i}\right|<\pi-2<1.16$ for $i=1,2,3$. This implies that the curves $C_{i}$ must be entirely inside the ellipse $\mathcal{E}$ with foci $a$ and $b$ and major axis of length 1.16. The length of the semi-major axis of $\mathcal{E}$ is $A=\frac{1.16}{2}=.58$ and the semi-minor axis is $B=\sqrt{.58^{2}-.5^{2}}<.294$. The circumference $\ell$ of $\mathcal{E}$ is less than $\ell \leq \sqrt{2} \pi \cdot \sqrt{A^{2}+B^{2}}<2.9$. Here we used the upper bound on the perimeter of an ellipse from Proposition 3 of Jameson [48].

Now cut $\mathcal{E}$ with vertical lines through $a$ and 6 and let $D_{1}$ be the path starting at $a$ and moving vertically up to the bordering arc of $\mathcal{E}$ at point $a_{1}$ and then proceed on the arc until $b_{1}$, where the vertical line passing through 6 intersects $\mathcal{E}$ and then move to 6 in the vertical direction (see Fig. 3). We have a similar path $D_{2}$ from $a$ through $a_{2}$ and $b_{2}$ to 6 in the part of $\mathcal{E}$ below the line of $a b$. The total length of $D_{1}$ and $D_{2}$ is at most $\ell<3$; hence they cannot give a correct solution to SDP. Therefore, there exists a point $z \in Z$ on the plane and points $p \in D_{1}$ $q \in D_{2}$ such that the disk $\mathcal{D}$ of radius $r$ centered at $z$ contains $p$ and $q$ in its interior.

It suffices now to exhibit a point $p^{\prime}$ on the upper arc $a_{1} b_{1}$ of $\mathcal{E}$ and $q^{\prime}$ of the lower arc $a_{2} b_{2}$ of $\mathcal{E}$ such that both $p^{\prime}$ and $q^{\prime}$ are in the interior of $\mathcal{D}$. Then the line segment $p^{\prime} q^{\prime}$ intersects all of the curves $C_{i}$ and stays in the interior of $\mathcal{D}$, which implies that these curves do not protect against failure at $z$.

To establish the existence of $p^{\prime}$, we consider $p$. If $p$ is on the arc $a_{1} b_{1}$ of $\mathcal{E}$, then $p^{\prime}=p$ is a correct choice. Suppose now that $p$ is on a vertical part of $D_{1}$. Wlog we assume that it is on the vertical part $a a_{1}$. Using the fact that $a$ is not in the interior of $\mathcal{D}$, we see that $z$ must lie over the line of $a b$. Also, $z$ is to the right of the line of $a a_{1}$, because the interior of $\mathcal{D}$ intersects $D_{2}$. The distance of $z$ from $a a_{1}$ is at most 0.51 , hence it is to the left of the line of $b b_{1}$. Suppose first 


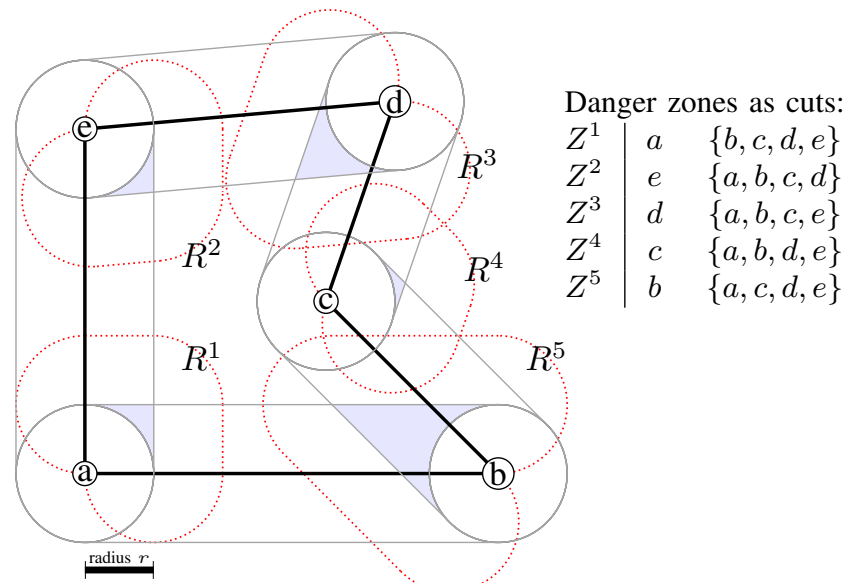

(a) The danger zones are colored blue, and red dotted lines show the border of the zones after offsetting by $r$.

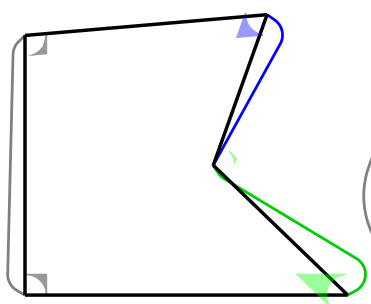

(b) Solution for small $r$

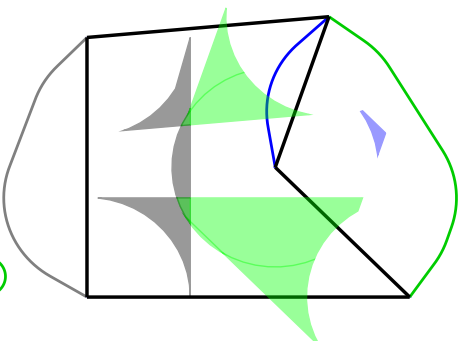

(c) Solution for large $r$
Fig. 5. An example network of 5 nodes and 5 links.

that $z \in \mathcal{E}$. Consider now the highest point $h$ of $\mathcal{D}$. This $h$ is outside $\mathcal{E}$ because $|h z|=r>0.4>B$. Now $p^{\prime}$ may be the intersection point of $h z$ and the arc of $\mathcal{E}$. Clearly, $p^{\prime}$ is to the right of $a a_{1}$ and is to the left of $b b_{1}$, and over $a b$, as the whole segment $h z$ is in these half planes.

A similar consideration shows that if $z \notin \mathcal{E}$, then the intersection of the segment $p z$ with the border of $\mathcal{E}$ gives a suitable $p^{\prime}$.

A suitable point $q^{\prime}$ can be constructed by using $q$ in an analogous way. This finishes the proof.

\section{Heuristic Algorithm for Two Curve SDP Solution}

Based on the above, we implemented the following straightforward heuristic to compute $C_{1}$ and $C_{2}$ for $l=2$ in the case of a general $Z$. We divide $Z$ by the line of $a b$ into $Z_{1}$ and $Z_{2}$, and curve $C_{1}$ is at distance $r$ from $Z_{1}$ and $C_{2}$ is at distance $r$ from $Z_{2}$. Finally, we compare the total length $\left|C_{1}\right|+\left|C_{2}\right|$ with the single curve solution $|C|$ which is of distance at least $r$ from $Z$.

The above heuristic is not optimal, even for a uniform cable cost. Fig. 4 shows an example shape of $Z$, where dividing $Z$ by the line through points $a$ and $b$ does not give the optimal solution (blue curves). Then $Z_{1}$ and $Z_{2}$ are the optimal division of $Z$ into two disjoint areas, which are shaded with two different blue colors. Because of the specific shapes of the danger zones, the heuristic managed to find the optimal solution in all examined problem instances. The GAP between the costs in Fig. 4 is only $>8 \%$. We leave for future research

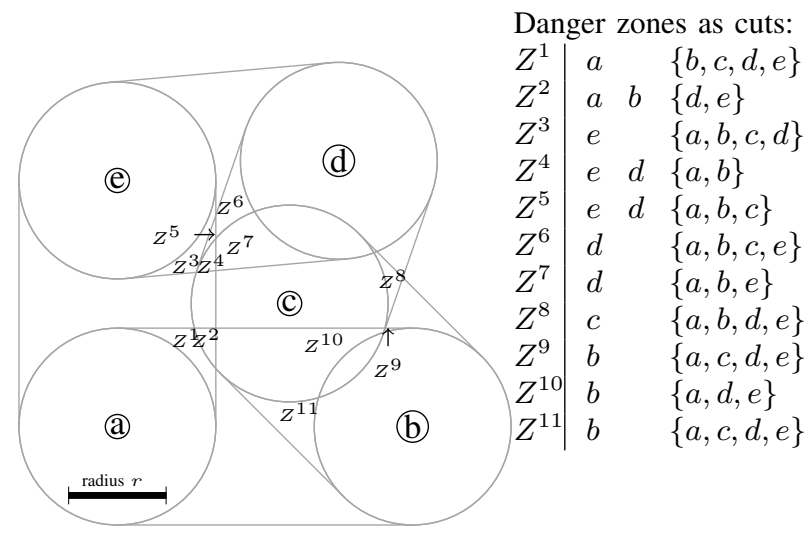

(a) The danger zones of the small network with larger radius.

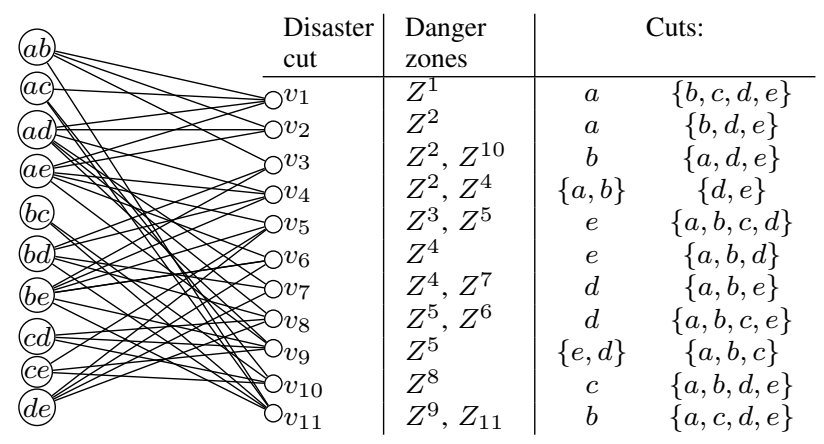

(b) The bipartite auxiliary graph for the optimization.

Fig. 6. The 5-node example network with larger radius.

to further investigate Subproblem 2, including the case of general cable installation cost.

\section{Algorithmic Framework for the Geometric Network Augmentation (GNA) Problem}

Before we provide the problem formulation, let us discuss a small, five node ring network shown in Fig. 5. Using an argument similar to the one used at the single edge network example, we can draw the danger zones, which are epicenters of failures that will result in isolated network components. We have five such danger zones, denoted by $Z^{1}, \ldots, Z^{5}$, each defining a cut in the graph. For example a failure with epicenter in $Z^{1}$ would isolate a network with node $a$ from $\{b, c, d, e\}$. To protect the network against a failure of an epicenter in $Z^{1}$, we need to add a new link, whose distance to the danger zone $Z^{1}$ is at least $r$. We draw the boundaries of the $r$-inflated danger zones by red dotted lines, there are 5 such areas denoted by $R^{1}, \ldots, R^{5}$, as shown in the table on the right of Fig. 5a.

Fig. 5b shows the optimal solution for the problem of Fig. 5a. Each new edge has a unique color, and we color the danger zone according to the edge it is protected by. Recall that there are 5 danger zones, and the new gray edge protects $Z^{1}$ and $Z^{2}$, the blue $Z^{3}$, and the green $Z^{4}$ and $Z^{5}$. Here every danger zone is protected by a single curve.

Fig. 6 a shows how complicated the situation becomes for larger $r$. Here we have 11 danger zones, and some danger 


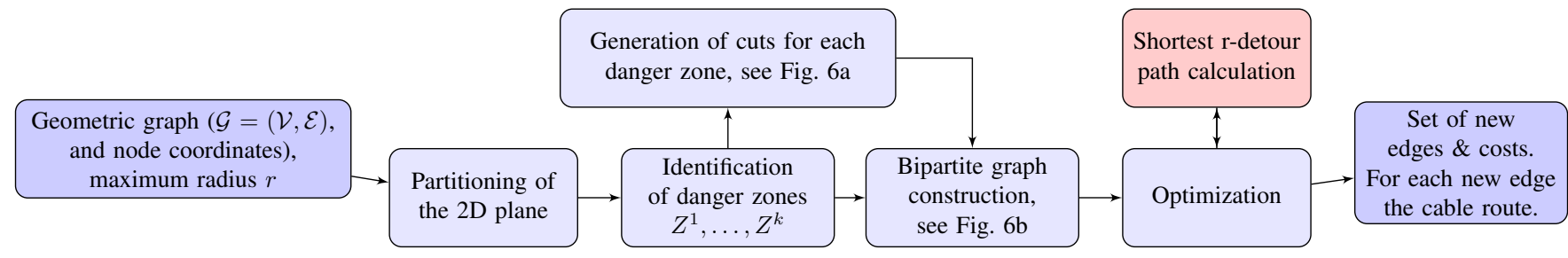

Fig. 7. Flowchart for the generic steps required to solve the GNA problem. Note that the sub-problem described in sec. III (colored red on the figure) must be solved for many configurations during the optimization.

zones cut the graph into three components, for example, $Z^{2}$. The optimal solution is shown on Fig. 5c, where we have 3 new edges: the black, blue, and green, with their corresponding danger zones colored accordingly. The danger zone $Z^{2}$ is plotted in blue-gray mixed color because the corresponding three components are connected with two edges: the gray and blue.

\section{A. Non-linear Mathematical Program Formulation}

Let us formulate the problem as a mathematical program. See Fig. 7 for the flowchart of the whole optimization process. First, we draw a radius $r$ circle around each node of the graph. For each edge, we also add two parallel line-segments with the same length and distance $r$ from the edge on both sides, see Fig. 6. These curves will divide the plane into faces, and their collection is called an arrangement. Computing an arrangement of a set of (simple) geometric objects (e.g., disks, rectangles) in the plane is a well-studied problem, and the number of faces is at most $O\left(\mathrm{~m}^{2}\right)$, see also [49]. Every point of a face selected as an epicenter of a disk failure hits the same set of edges and nodes, called residual network $\mathcal{G}_{c}$. The faces where the corresponding residual network is not connected, are the danger zones $Z^{1}, \ldots, Z^{k}$.

Now we can formulate a discrete optimization problem. First we will evaluate each face, compute the residual network $\mathcal{G}_{c}$ and its number of components $t$. If $t=2$ we call them disaster cuts. Otherwise, if the danger zones separate the graph into $t \geq 3$ components (e.g. $Z^{2}$ on Fig. 6a), we will convert it into a set of disaster cuts. In this case we add $2^{t-1}-1$ disaster cuts: every combination of two component cuts: for example danger zone $Z^{2}$ with 3 components $a|b| d e$ on Fig. 6a should be transformed into 3 disaster cuts: $a|b d e, b| a d e$ and $a b \mid d e$. Note that, a danger zone with four components $A|B| C \mid D$ would be transformed into the following 7 disaster cuts: $A \mid B C D$, $B|A C D, C| A B D, A B C|D, A B| C D, A C \mid B D$ and $A D \mid B C$. Finally, we merge any two disaster cuts if they result in the same set of nodes, see the table on Fig. 6b. Let $l$ be the number of disaster cuts after merging the list. It is 11 for the example of Fig. 6.

Finally, we can formulate the problem as a (nonlinear) optimization problem as follows: we define a bipartite graph $\left(V_{n n}, V_{z}, E_{z}\right)$ as shown in Fig. 6b. Each node in $V_{z}$ corresponds to a disaster cut, thus $\left|V_{z}\right|=l$. Each node in $V_{n n}$ corresponds to a distinct node-pair $a, b$ of $V$, i.e. $a \neq b$. Note that we can erase those node-pairs that cannot protect any disaster cut. We have also $\left|V_{n n}\right| \leq \frac{n(n-1)}{2}$. As for the edges, we connect every node $v_{a b} \in V_{n n}$ and $v_{i} \in V_{z}$, if connecting node $a$ with node $b$ would connect the two sides of the disaster cut $v_{i}$.

For each edge between $v_{i} \in V_{z}$ and $a b \in V_{n n}$ we have the following binary variable:

$$
x_{i}^{a b}= \begin{cases}1 & \text { add link }(\mathrm{s}) \text { between } a \text { and } b \text { that connects } \\ & \text { the two sides of disaster cut } v_{i}, \\ 0 \quad & \text { otherwise }\end{cases}
$$

The only constraints we have that every danger cut must be protected:

$$
\sum_{\forall\left(v_{a b}, v_{i}\right) \in E_{z}} x_{i}^{a b} \geq 1 \quad i=1, \ldots, l .
$$

The objective function is as follows:

$$
\text { minimize: } \sum_{\forall v_{a b} \in V_{n n}}\left|\mathcal{C}_{Z_{a b}}^{a b}\right|,
$$

where $Z_{a b}$ denotes the union of danger zones for each $v_{i}$ such that $x_{i}^{a b}=1$. It is the cost of the shortest curve(s) between nodes $a$ and $b$ protecting a set of danger zones, computed by the heuristic presented in Sec. III.

With the above problem formulation, we have divided GNA into a master problem and separate sub-problems. The subproblems are how to compute $\mathcal{C}_{Z}^{a b}$, i.e., the minimum cost cable routes between given end nodes and danger zones presented in Sec. III. The master problem is considered in this section, it is to determine how to select the end nodes of the new edges and decide which new edge protects each danger zone. Note that since $\mathcal{C}_{Z}^{a b}$ is solved with a heuristic, the optimal solution of the mathematical problem may not be the global optimum for the GNA problem.

\section{B. Greedy Baseline Heuristic (GBH)}

Our first heuristic to solve the master problem is a greedy algorithm based on a greedy iterative search. It takes advantage of Lovász's approach for minimum set cover, where the items correspond to disaster cuts, and the sets correspond to new edges. However, in our problem, we have a sophisticated cost function of the sets covering the items, i.e., $\mathcal{C}_{Z}^{a b}$.

The heuristic algorithm chooses the most feasible-looking edge from the left side of the bipartite graph in each iteration. Other factors can help choose the next edge, for example, the 
average cost per protected cut, or the number of cuts protected by the edge, but in this study, the cheapest edge is selected.

After the selection is made, the algorithm deletes the selected edge with all its neighboring cuts from the right side (as they are now protected). It continues this choose-delete loop until we are left with no cuts in the bipartite disaster graph. The selected edges will protect every cut since the condition for stopping is that there are none of them left unprotected, but this process may cause significant overhead.

\section{Integer Linear Program (ILP)}

The problem formulated by Eq. (1) and (2) has a nonlinear cost function (3). Next we reformulate the nonlinear optimization problem into an Integer Linear Program (ILP). The ILP will have an exponential number of working variables. Roughly speaking through a new set of 0,1 -variables $z$ the formulation will contain all executions of $\mathcal{C}_{Z}^{a b}$. Note that the possible inputs of $\mathcal{C}_{Z}^{a b}$ are the node pair $a b$, and a set of danger zones corresponding to the edges adjacent with $v_{a b}$ in the bipartite graph, i.e., subsets of $N\left(v_{a b}\right)$. Let $S^{a b}$ denote the set of every possible nonempty subsets of edges connected to node $v_{a b} \in V_{n n}$ in the bipartite graph, in other words the power set $P\left(N\left(v_{a b}\right)\right)$ minus the empty set. Note that there are $2^{\left|N\left(v_{a b}\right)\right|}-1$ such sets. Let $S=\cup_{v_{a b} \in V_{n n}} S^{a b}$ denote the union of them for all nodes in $V_{n n}$. We have a total of $|S|=\sum_{a b \in V_{n n}}\left|S^{a b}\right|$ binary variables, we denote them by $z_{s}$ for $s \in S$. In other words, each variable $z_{s}$ corresponds to a node pair $a b$ and a (sub)set of danger zones (denote it by $Z_{s}$ ) it can protect. The linear objective function is

$$
\text { minimize: } \sum_{\forall v_{a b} \in V_{n n}} \sum_{\forall s \in S^{a b}}\left|\mathcal{C}_{Z_{s}}^{a b}\right| \cdot z_{s} \text {. }
$$

The constraints are

$$
\sum_{\forall s \in S \mid i \in Z_{s}} z_{s} \geq 1 \quad \forall v_{i} \in V_{z}
$$

where $i \in Z_{s}$ means the danger zone corresponding to $v_{i}$ is part of the danger zone set $Z_{s}$.

Roughly speaking, the reformulated ILP has variables that correspond to edge sets in $E_{z}$, instead of having a variable for each independent edge of $E_{z}$. Therefore this problem formulation is significantly larger than the one in Sec. IV-A; however, it has a linear objective function.

Note that in general, solving an ILP can be computationally hard; however, in our case, it takes significantly less time than to run the SDP heuristic many times. The latter task is necessary when formulating the ILP. Later in the experimental section, we will show that the number of danger zones is small in practice. Further note that the ILP solution is not guaranteed to be an optimal solution to the proposed GNA problem since it uses the results of a heuristic algorithm for the SDP.

\section{Dual LP Heuristic (DLPH)}

Next, we introduce a Dual LP Heuristic (DLPH) to replace the ILP in the previous scheme for achieving a reduced number of variables and thus reduced running time. It is clear that the dual LP has only $\left|V_{z}\right|$ variables, and we use only a small portion of constraints of the dual of the previous ILP formulation, thus yielding way less computation complexity. Note that the weak duality theorem does not apply in this case, and thus the dual LP does not provide a lower bound for the ILP.

Next, we provide the dual LP formulation of the problem. Let $y_{i}$ denote a variable assigned to each disaster cut $v_{i} \in V_{z}$, which is intuitively the cost of protecting disaster cut $i$. The goal is to

$$
\text { maximize: } \sum_{v_{i} \in V_{z}} y_{i} \text {. }
$$

The constraints are given in the following

$$
\sum_{\forall v_{i} \in V_{z} \mid i \in Z_{s}} y_{i} \leq\left|\mathcal{C}_{Z_{s}}^{a b}\right| \quad \forall s \in S^{a b}, \forall v_{a b} \in V_{n n} .
$$

Note that $S$ is composed of the power sets of $N\left(v_{a b}\right)$ for $\forall v_{a b} \in V_{n n}$. To reduce the time we only generate one constraint for each $v_{a b}$, corresponding to set $N\left(v_{a b}\right)$. Finally, we transform the dual solution into a binary primal one, where we count on the fact that each dual constraint shall correspond to a variable in the primal. More precisely, if the dual constraint is tight, the corresponding primal variable is set to 1 . A constraint is called tight if the inequality holds with equality in the optimal solution.

The above heuristic can also be explained just using the primal problem. In this case, we solve a traditional set cover problem where the items correspond to disaster cuts, and the sets correspond to possible new edges. The cost of a set corresponding to the new edge $a b$ equals to $\left|\mathcal{C}_{N\left(v_{a b}\right)}^{a b}\right|$. In other words, we limit the search space to the case where each new edge $a b$ protects every danger cut where $a$ and $b$ are on the opposite sides of the cut.

\section{EXPERIMENTAL RESULTS}

In this section, we present numerical experimental results by using real backbone network topologies to demonstrate the merits of the proposed three heuristics: GBH (Sec. IV-B), ILP (Sec. IV-C), and DLPH (Sec. IV-D). We investigate various aspects of system performance, e.g., how the given disk radius values $r$ or the network density impacts the overall cost and running time.

Our algorithms are implemented ${ }^{1}$ in Python with binding to two open-source $\mathrm{C}++$ implementations: the 2D Arrangements package from the CGAL library ${ }^{2}$ for computing divisions of the plane, and the SIG class toolkit ${ }^{3}$ for offsetting polygons and geometric Dijkstra algorithm with a uniform cost. We used the SDP heuristic of Sec. III-C to compute the multi-curve solution. For practical considerations, we ignore extremely narrow danger zones.

\footnotetext{
${ }^{1}$ All experimental results with additional networks, as well as the source code are available online https://github.com/hajduzs/netext including the network typologies and the obtained list of curves.

${ }^{2}$ https://www.cgal.org/

${ }^{3}$ https://bitbucket.org/mkallmann/sig/wiki/Home
} 


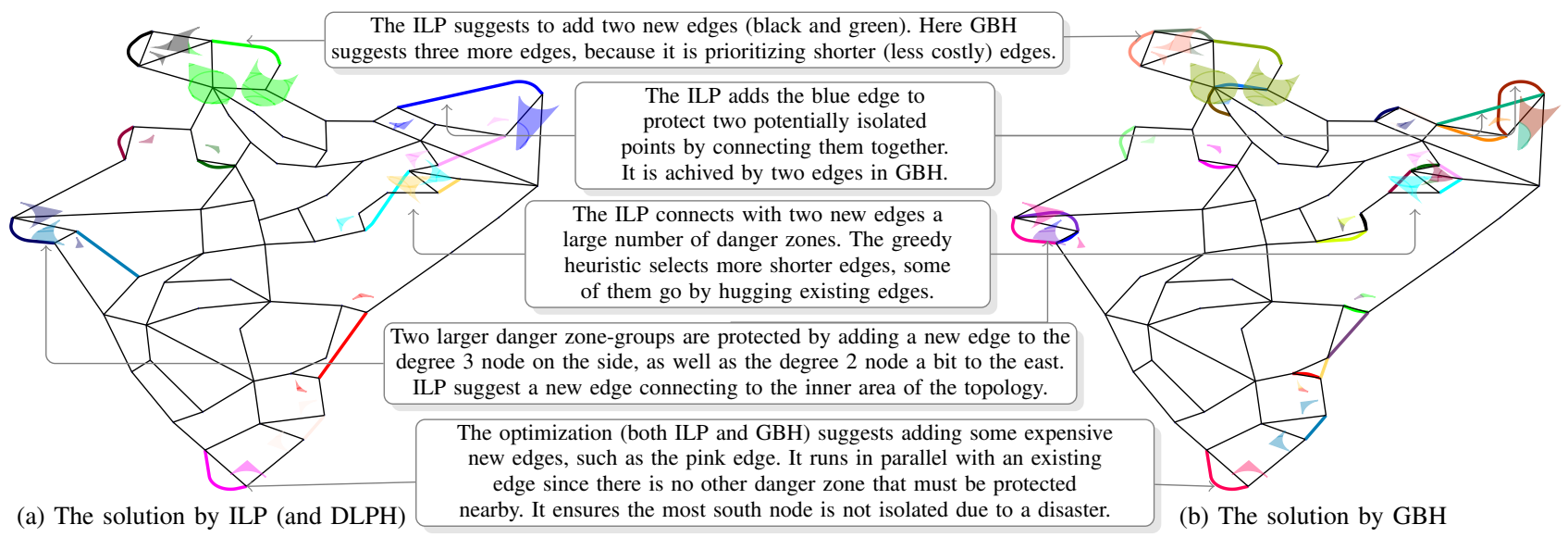

Fig. 8. GNA Problem Solution for Tata (India) backbone network $(r=80 \mathrm{~km})$.

\section{A. ILP vs GBH for India (Tata) Backbone Network}

First we present the results of using ILP and GBH on the Tata (India) backbone network, respectively, aiming to highlight the performance gap between the two schemes. The goal of the optimization is to protect disasters of radius $r=80 \mathrm{~km}$. A few recent examples of natural disasters with affected areas of larger radius are the Uttarakhand Flash Floods ${ }^{4}$ in 2013, the Kashmir Floods ${ }^{5}$ in 2014, and the tropical Cyclone Amphan ${ }^{6}$ in 2020.

Fig. 8 shows the new edges suggested by the ILP as well as the GBH algorithm. The danger zones and the corresponding new edges are drawn with the same color. In total, 87 danger zones have to be protected, which results in 49 cuts.

The ILP solution in Fig. 8a shows the enhancement of the India network to be $r=80 \mathrm{~km}$ disaster-resilient. It requires 13 new edges with a total length of $3425 \mathrm{~km}$, i.e., $17 \%$ additional cable is necessary. In the rest of the section, for the cost comparison, we utilize the metric $C^{\%}$, which is the new edge length divided by the total length (given in \%).

As a comparison, GBH requires 27 new edges with a total length of $5085 \mathrm{~km}$, meaning $C^{\%}=25 \%$ additional cable length is needed, i.e., when utilizing the heuristic $41 \%$ extra cable length is needed. However, the running time (denoted as $t$ ) of the ILP is $88 \mathrm{~min}$ compared with $18 \mathrm{~min}$ by the GBH. Nonetheless, the GBH solutions result in approximately twice more edges (some even cut through existing edges see Fig. $8 b$ ), which are not considered useful in quality. It comes from the greedy nature of GBH, where the shortest (less costly) edges are selected at the beginning, leading to a larger total length at the end, as shown in the examples of Fig. 8.

Every danger zone is protected by a single curve SDP solution. Note that many of the short edges run almost parallel to the existing ones next to degree 2 nodes. It is a consequence of uniform cable installation costs. In the case of general cable cost, we could set the cable installation cost small if the cables

\footnotetext{
$4_{\text {https://reliefweb.int/sites/reliefweb.int/files/resources/IND_UttarandLocationmap_130628.pdf }}$

5 https://sphereindiablog.files.wordpress.com/2014/09/04jkflood_relief-camps_jammu-division_sept.jpg

$6_{\text {https://reliefweb.int/sites/reliefweb.int/files/resources/Sitrep-Cyclone-AMPHAN-3.pdf }}$
}

should be deployed along with existing cables, which would avoid using these parallel new edges.

In general, we can observe that most of the edges are added due to the 2 degree nodes, especially outside the network core. As expected, all the new edges follow the single edge solution, and all the cuts divide the network into two components. The relationship between the metrics is further discussed in the performance evaluation section.

\section{B. Performance Evaluation}

For the performance evaluation of the algorithms, we selected eleven additional topologies and analyzed the results for various radii. The summary of the experimental results is shown for each network in Table I (for $r=40 \mathrm{~km}$ and $r=80 \mathrm{~km})$ and some of the main tendencies are displayed in Fig. 9.

In Table I we can observe that the cost of the new edges by the ILP is below $17 \%$ in each scenario and, on the average it is $9.4 \%$ for $r=40 \mathrm{~km}$ and $10.8 \%$ for $r=80 \mathrm{~km}$. For simpler cases, GBH yields a solution with performance very close to the ILP, while much outperformed in more complicated cases. With the GBH heuristic, the cost was always below 25\%, and the GAP was at most $63 \%$. Besides, Table I shows that with the number of danger zones between 3 and 116, maximally 46 cuts are in place. As expected, the larger networks (i.e., with more nodes and edges), there are more danger zones and cuts in the networks, leading to a larger number of constraints and thus higher complexity. Nonetheless, the total cost is related to neither the number of danger zones and thus the number of cuts, nor the number of new edges required in a solution (which ranges from 2 to even 14).

Fig. 9a shows the runtime and solution quality tradeoff for the three algorithms GBH, DLPH, and ILP. It is an average of $12 \times 20$ runs, where we have 12 networks, as shown in Table I, and radii $r=5,10, \ldots, 100 \mathrm{~km}$. The runtime of each algorithm for every network can be seen in Table I for $r=40,80 \mathrm{~km}$. The chart shows that the ILP has the longest average runtime (i.e., 5 minutes). On average, DLPH is faster 
TABLE I

SUMMARY OF THE EXPERIMENT RESULTS FOR VARIOUS PRACTICAL NETWORK TOPOLOGIES. THE FOLLOWING METRICS ARE SHOWN: THE NUMBER OF DANGER ZONES $(|Z|)$ AND DANGER CUTS $\left(\left|V_{Z}\right|\right)$, THE SIZE OF THE ILP IN TERMS OF VARIABLES (|EQ $\left.\mid\right)$, THE GAP (GAP) TO THE OPTIMAL SOLUTION OF SEC. IV-A, THE TOTAL LENGTH OF THE NEW EDGES DIVIDED BY THE TOTAL CABLE LENGTH IN PERCENT $(C \%)$, THE NUMBER OF NEW EDGES ADDED $\left(\left|E_{n}\right|\right)$ AND THE RUNNING TIME $(t)$. THE COLUMNS WITH THE SOLUTIONS OF DLPH ARE OMITTED AS THEY ARE THE SAME AS FOR ILP.

\begin{tabular}{|c|c|c|c|c|c|c|c|c|c|c|c|c|c|c|c|c|c|c|c|c|c|c|c|c|c|}
\hline \multicolumn{4}{|c|}{ Backbone network topology } & \multicolumn{11}{|c|}{$r=40 \mathrm{~km}$} & \multicolumn{11}{|c|}{$r=80 \mathrm{~km}$} \\
\hline & & & total edge & & hger & & II & & & DLPH & & $\mathrm{GH}$ & & & & nger & & Il & & & DLPH & & & $\mathrm{BH}$ & \\
\hline Name & & & length $(\mathrm{km})$ & $|Z|$ & $\left|V_{Z}\right|$ & |Eq $\mid$ & $C^{\%}$ & $\mid E_{n}$ & $t(\mathrm{~s}$ & $t(\mathrm{~s})$ & GAP & $C^{\%}$ & $\left|E_{n}\right|$ & $t(\mathrm{~s})$ & $|Z|$ & $V_{Z}$ & $|\mathrm{Eq}|$ & $C^{\%}$ & $\left|E_{n}\right|$ & $t(\mathrm{~s})$ & $t(\mathrm{~s})$ & GAP & $C^{\%}$ & $\left|E_{n}\right|$ & $t(\mathrm{~s})$ \\
\hline Gridnet (US) [50] & 9 & 20 & 42635 & 12 & 6 & 108 & 10 & 2 & 4.90 & 3.71 & 4.85 & 10 & 4 & 1.02 & 10 & 5 & 67 & 10 & 2 & 3.65 & 3.20 & 35 & 14 & 4 & 0.82 \\
\hline GlobalCenter (US) [50] & 9 & 36 & 571 & 5 & 3 & 27 & 4.07 & 3 & 4.61 & 4.51 & 0 & 4.07 & 3 & 0.19 & 7 & 4 & 38 & 5.08 & 3 & 4.48 & 4.21 & 1.47 & 5.16 & 4 & 0.29 \\
\hline Pan-European [22] & 16 & 22 & & 4 & 4 & 66 & 16 & 4 & 3.97 & 3.63 & 0 & 16 & 4 & 0. & 6 & 5 & 100 & 22 & 4 & 3.87 & 3.81 & 6.55 & 24 & 5 & $\overline{0.59}$ \\
\hline Electric Lightwave [50] & 20 & 30 & 236 & 10 & 6 & 148 & 5.43 & 4 & 4.43 & 3.8 & 22 & 6.66 & & & 11 & & 304 & 5.86 & 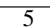 & 6.53 & 4.47 & 34 & 7.87 & 7 & $\overline{3.02}$ \\
\hline COST & 22 & 45 & & 5 & & 115 & 12 & 4 & 4.02 & 4.17 & 18 & 15 & 5 & & 5 & & 115 & 13 & & 3.71 & 3.79 & 17 & 15 & 5 & 0.53 \\
\hline US nation & 24 & 42 & & 6 & & 153 & 6.17 & 4 & 3.69 & 3.59 & 29 & 8.02 & 6 & 0.70 & 6 & & 153 & 6.51 & & 4.06 & 3.74 & 27 & 8.32 & 6 & 0.66 \\
\hline France & 25 & 45 & 11487 & 58 & 17 & 2251 & 9.61 & 7 & 558 & 40 & 39 & 12 & 11 & 115 & 116 & 35 & 59332 & 14 & $\varepsilon$ & 55128 & 104 & 63 & 23 & 18 & 764 \\
\hline Darkstrand (USA) [50] & 28 & 31 & 14411 & 5 & 5 & 145 & 8.02 & 3 & 3.65 & 3.50 & 49 & 11 & 5 & 0.58 & 6 & 6 & 205 & 9.23 & 3 & 3.66 & 3.52 & 38 & 12 & 5 & 0.65 \\
\hline Nobel (EU) [51] & 28 & 41 & & 5 & 5 & 145 & 11 & 4 & 4.28 & 4.44 & 0 & 11 & 4 & 0.60 & 4 & 4 & 114 & 12 & 4 & 3.74 & 4.53 & 0 & 12 & 4 & 0.53 \\
\hline Pioro (random) [51] & 40 & 89 & 4202 & 9 & 8 & 340 & 4.92 & 6 & 4.66 & 4.00 & 17 & 5.79 & 7 & 1.63 & 16 & 11 & 655 & 6.20 & 6 & 10 & 6.24 & 14 & 7.08 & 7 & 5.04 \\
\hline Telia (USA) [52] & 44 & 65 & 30295 & 26 & 16 & 1078 & 13 & 11 & 27 & 10 & 31 & 18 & 15 & 77 & 34 & 17 & 1783 & 14 & 9 & 55 & 13 & 41 & 20 & 15 & 108 \\
\hline Tata (India) [53] & 70 & 97 & 20047 & 40 & 27 & 6468 & 13 & 14 & 226 & 31 & 37 & 18 & 21 & 445 & 87 & 49 & 56149 & 17 & 13 & 5290 & 71 & 41 & 25 & 27 & 1096 \\
\hline
\end{tabular}

than $\mathrm{GBH}$ because it requires less computation of $\mathcal{C}_{Z}^{a b}$, which is the dominant factor if the radius $r$ is large and there are more danger zones close to each other in place. Nevertheless, DLPH finds a solution with a slightly higher cost than ILP. Fig. 9b shows the aggregated relative cost versus the radii of each algorithm. DLPH finds the same solution as the ILP for $r \leq 80 \mathrm{~km}$, and there is little difference for larger radii where we have a more complex arrangement structure due to a large number of overlapping geometric objects, see also Fig. 6. Overall, DLPH outperforms both ILP and GBH when optimizing jointly for cost and running time.

In Fig. 9b, it can be observed that the ILP solution scales very well with the radii, where the protection costs increase linearly with a small slope as a function of $r$. This is also attested by the fact that doubling the radii (from $40 \mathrm{~km}$ to $80 \mathrm{~km}$ ) only yields around $1 \%$ increase in cost, as shown in Table I.

Fig. 9c shows the average number of new edges for all networks, which increases modestly as the radius $r$ increases. Specifically, we need an average of 4.7 new edges to protect disasters at $r=20 \mathrm{~km}$ and slightly more 5.2 new edges at $r=100 \mathrm{~km}$. Note that the GBH solution yields more short links, where altogether $1 \%$ are two curve SDP solutions (15 out of 1169 new edges); while the DLPH solution yields only $0.1 \%$ ( 1 out of 616 new edges) as it tends to select longer edges than GBH. In the case of the ILP, on the other hand, since every danger zone was protected by a single curve (there were 441 new edges in total), thus rarely yielding a two curve SDP solution. Finally, Fig. 9d presents how the average numbers of danger zones and cuts scale as a function of the radius $r$. It shows that the number of cuts increases more slowly than that of the danger zones.

\section{CONCLUDING REMARKS}

In this paper, we investigate the augmentation of the topology of a backbone network with new cables to maintain network connectivity under regional failures. A novel design framework, namely Geometric Network Augmentation (GNA), is introduced, which targets to identify a set of cable routes that can protect each danger zone. We divide the overall problem into a master problem and a separate sub-problem. The sub-problem determines the optimal cable route(s) for a given pair of nodes, while the master problem aims to select the end nodes for allocating the new cable routes and decide which new cable route protects each danger zone. The proposed solutions, namely ILP, GBH, and DLPH, are examined via extensive simulation in a number of practical network topologies. Our findings from the simulations are summarized as follows.

- The nodes with outgoing connections in diversified directions (e.g., the nodes in the geometrical center of the network) rarely require additional protection edges. On the other hand, the degree 2 (and 1) nodes connected with an acute angle are often in need of protection, where a potential disaster could make the nodes isolated.

- High computation complexity usually arises due to the network regions with a large number of highly connected nodes, where a disk failure cuts the graph into several components, leading to an exponential number of 2-cuts.

- The ILP yields solutions with the best quality among the three proposed algorithms at the expense of the longest running time. DLPH, on the other hand, is observed to achieve the best tradeoff between the running time and cost. It is recommended for practical application scenarios.

\section{ACKNOWLEDGMENT}

We would like to thank Marcelo Kallmann for the guide on the SIG code; Amaro de Sousa, Martin Zachariasen, David Hay, Balázs Vass for the inspiring discussions.

The research leading to these results was partially supported by the High Speed Networks Laboratory (HSNLab). This article is based on work from COST Action CA15127 ("Resilient communication services protecting end-user applications from disaster-based failures" - RECODIS), supported by COST (European Cooperation in Science and Technology). Projects no. 123957, 129589, 124171, 134604 and 128062 have been implemented with the support provided from the National Research, Development and Innovation Fund of Hungary, financed under the FK_17, KH_18, K_17, FK_20 and K_18 


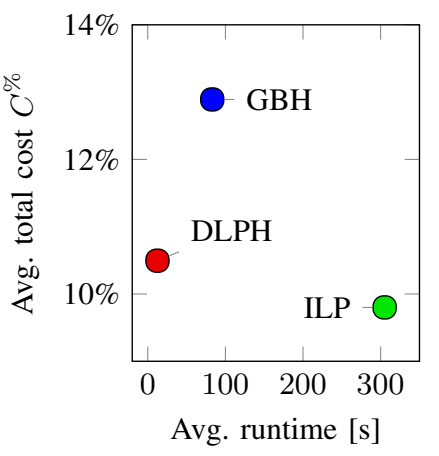

(a) Runtime vs cost

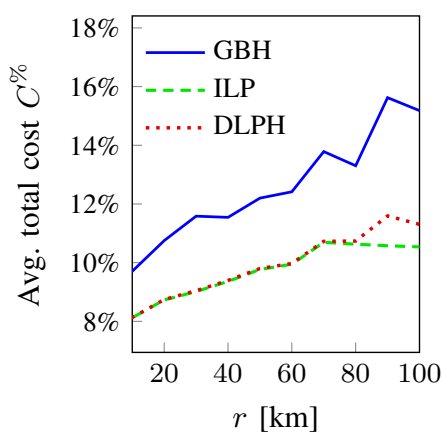

(b) The cost of the solution

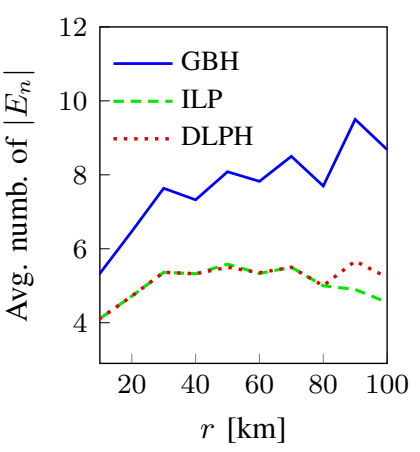

(c) The number of new edges

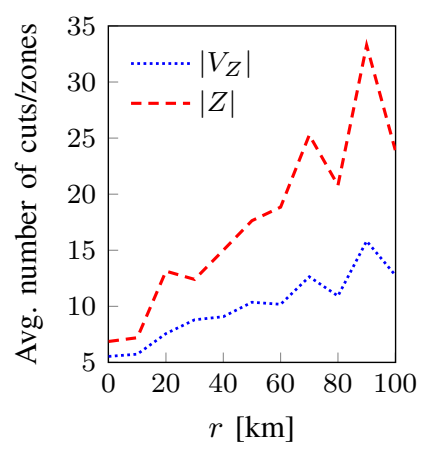

(d) \# danger zones $(|Z|)$ and cuts $\left(\left|V_{Z}\right|\right)$

Fig. 9. The mean performance for all networks of Table I for radii $r=5,10, \ldots, 100$.

funding schemes respectively. The research reported in this paper and carried out at the BME was supported by the "TKP2020, Institutional Excellence Program" of the National Research Development and Innovation Office in the field of Artificial Intelligence (BME IE-MI-SC TKP2020). The work of Lajos Rónyai was supported in part by the NKFIH/OTKA under Grant K115288, and by the Hungarian Ministry of Innovation and the National Research, Development and Innovation Office within the framework of the Artificial Intelligence National Laboratory Programme.

\section{REFERENCES}

[1] S. Neumayer, G. Zussman, R. Cohen, and E. Modiano, "Assessing the vulnerability of the fiber infrastructure to disasters," Networking, IEEE/ACM Transactions on, vol. 19, no. 6, pp. 1610-1623, 2011.

[2] O. Gerstel, M. Jinno, A. Lord, and S. B. Yoo, "Elastic optical networking: A new dawn for the optical layer?" Communications Magazine, IEEE, vol. 50, no. 2, pp. s12-s20, 2012.

[3] M. F. Habib, M. Tornatore, M. De Leenheer, F. Dikbiyik, and B. Mukherjee, "Design of disaster-resilient optical datacenter networks," Journal of Lightwave Technology, vol. 30, no. 16, pp. 2563-2573, 2012.

[4] J. Heidemann, L. Quan, and Y. Pradkin, A preliminary analysis of network outages during hurricane Sandy. University of Southern California, Information Sciences Institute, 2012.

[5] F. Dikbiyik, M. Tornatore, and B. Mukherjee, "Minimizing the risk from disaster failures in optical backbone networks," Journal of Lightwave Technology, vol. 32, no. 18, pp. 3175-3183, 2014.

[6] I. B. B. Harter, D. Schupke, M. Hoffmann, G. Carle et al., "Network virtualization for disaster resilience of cloud services," Communications Magazine, IEEE, vol. 52, no. 12, pp. 88-95, 2014.

[7] X. Long, D. Tipper, and T. Gomes, "Measuring the survivability of networks to geographic correlated failures," Optical Switching and Networking, vol. 14, pp. 117-133, 2014.

[8] B. Mukherjee, M. Habib, and F. Dikbiyik, "Network adaptability from disaster disruptions and cascading failures," Communications Magazine, IEEE, vol. 52, no. 5, pp. 230-238, 2014.

[9] R. Souza Couto, S. Secci, M. Mitre Campista, K. Costa, and L. Maciel, "Network design requirements for disaster resilience in iaas clouds," Communications Magazine, IEEE, vol. 52, no. 10, pp. 52-58, 2014.

[10] M. Van der Wee, S. Verbrugge, M. Tahon, D. Colle, and M. Pickavet, "Evaluation of the techno-economic viability of point-to-point dark fiber access infrastructure in europe," IEEE/OSA Journal of Optical Communications and Networking, vol. 6, no. 3, pp. 238-249, 2014.

[11] M. Maier, M. Herzog, M. Scheutzow, and M. Reisslein, "Protectoration: a fast and efficient multiple-failure recovery technique for resilient packet ring using dark fiber," Journal of lightwave technology, vol. 23, no. 10 , pp. 2816-2838, 2005.

[12] D. Tipper, "Resilient network design: challenges and future directions," Telecommunication Systems, vol. 56, no. 1, pp. 5-16, 2014.
[13] A. Pašić, R. Girão-Silva, B. Vass, T. Gomes, F. Mogyorósi, P. Babarczi, and J. Tapolcai, "Fradir-ii: An improved framework for disaster resilience," in Int. Workshop on Resilient Networks Design and Modeling (RNDM). IEEE, 2019, pp. 1-7.

[14] D. L. Msongaleli, F. Dikbiyik, M. Zukerman, and B. Mukherjee, "Disaster-aware submarine fiber-optic cable deployment for mesh networks," Journal of Lightwave Technology, vol. 34, no. 18, pp. 42934303, 2016.

[15] Q. Wang, J. Guo, Z. Wang, E. Tahchi, X. Wang, B. Moran, and M. Zukerman, "Cost-effective path planning for submarine cable network extension," IEEE Access, vol. 7, pp. 61 883-61 895, 2019.

[16] S. Verbrugge, D. Colle, P. Demeester, R. Huelsermann, and M. Jaeger, "General availability model for multilayer transport networks," in Proc. DRCN, Lacco Ameno, Italy, Oct. 16-19, 2005.

[17] D. Zhou and S. Subramaniam, "Survivability in optical networks," IEEE Network, vol. 14, no. 6, pp. 16-23, 2000.

[18] A. Somani, Survivability and traffic grooming in WDM optical networks. Cambridge University Press, 2006.

[19] S. Yang, S. Trajanovski, and F. Kuipers, "Availability-based path selection and network vulnerability assessment," Wiley Networks, vol. 66, no. 4, pp. 306-319, 2015.

[20] T. Gomes, J. Tapolcai, C. Esposito, D. Hutchison, F. Kuipers, J. Rak, A. De Sousa, A. Iossifides, R. Travanca, J. André et al., "A survey of strategies for communication networks to protect against large-scale natural disasters," in RNDM. IEEE, 2016, pp. 11-22.

[21] A. Mauthe, D. Hutchison, E. K. Çetinkaya, I. Ganchev, J. Rak, J. P. G. Sterbenz, M. Gunkel, P. Smith, and T. Gomes, "Disaster-resilient communication networks: Principles and best practices," in Resilient Networks Design and Modeling. IEEE, 2016, pp. 1-10.

[22] J. Tapolcai, L. Rónyai, B. Vass, and L. Gyimóthi, "Fast enumeration of regional link failures caused by disasters with limited size," IEEE/ACM Transactions on Networking, pp. 1-14, 2020.

[23] M. F. Habib, M. Tornatore, F. Dikbiyik, and B. Mukherjee, "Disaster survivability in optical communication networks," Computer Communications, vol. 36, no. 6, pp. 630-644, 2013.

[24] J. P. Sterbenz, E. K. Cetinkaya, M. A. Hameed, A. Jabbar, and J. P. Rohrer, "Modelling and analysis of network resilience," in Int. Conference on Communication Systems and Networks (COMSNETS). IEEE, 2011, pp. 1-10.

[25] M. Ashraf, S. Idrus, F. Iqbal, R. Butt, and M. Faheem, "Disaster-resilient optical network survivability: a comprehensive survey," in Photonics, vol. 5, no. 4. Multidisciplinary Digital Publishing Institute, 2018, p. 35.

[26] F. Iqbal and F. Kuipers, "On centrality-related disaster vulnerability of network regions," in Int. Workshop on Resilient Networks Design and Modeling (RNDM). IEEE, 2017, pp. 1-6.

[27] Y. Cheng, D. Medhi, and J. P. G. Sterbenz, "Geodiverse routing with path delay and skew requirement under area-based challenges," Networks, vol. 66, no. 4, pp. 335-346, 2015.

[28] A. de Sousa, D. Santos, and P. Monteiro, "Determination of the minimum cost pair of D-geodiverse paths," in Int. Conference on Design of Reliable Communication Networks (DRCN 2017), Munich, 2017.

[29] M. W. Ashraf, S. M. Idrus, F. Iqbal, and R. A. Butt, "On spatially disjoint lightpaths in optical networks," Photonic Network Communications, vol. 36, no. 1, pp. 11-25, Aug 2018. 
[30] D. Papadimitriou and B. Fortz, "Reliability-dependent combined network design and routing optimization," in 2014 6th International Workshop on Reliable Networks Design and Modeling, Nov 2014, pp. 31-38.

[31] B. Elshqeirat, S. Soh, S. Rai, and M. Lazarescu, "Topology design with minimal cost subject to network reliability constraint," IEEE Transactions on Reliability, vol. 64, no. 1, pp. 118-131, March 2015.

[32] F. Robledo, P. Romero, and M. Saravia, "On the interplay between topological network design and diameter constrained reliability," in Int. Conference on the Design of Reliable Communication Networks (DRCN), 2016, pp. 106-108.

[33] S. Neumayer, A. Efrat, and E. Modiano, "Geographic max-flow and mincut under a circular disk failure model," Computer Networks, vol. 77, pp. 117-127, 2015.

[34] H. Saito, "Spatial design of physical network robust against earthquakes," Journal of Lightwave Technology, vol. 33, no. 2, pp. 443-458, 2015.

[35] — - "Analysis of geometric disaster evaluation model for physical networks," IEEE/ACM Transactions on Networking, vol. 23, no. 6, pp. $1777-1789,2014$.

[36] C. Cao, M. Zukerman, W. Wu, J. H. Manton, and B. Moran, "Survivable topology design of submarine networks," Journal of Lightwave Technology, vol. 31, no. 5, pp. 715-730, 2013.

[37] W. Wu, B. Moran, J. H. Manton, and M. Zukerman, "Topology design of undersea cables considering survivability under major disasters," in Int. Conf. on Advanced Information Networking and Applications Workshops. IEEE, 2009, pp. 1154-1159.

[38] A. Sen, S. Murthy, and S. Banerjee, "Region-based connectivity-a new paradigm for design of fault-tolerant networks," in Int. Conference on High Performance Switching and Routing. IEEE, 2009, pp. 1-7.

[39] Y. Awaji, H. Furukawa, S. Xu, M. Shiraiwa, N. Wada, and T. Tsuritani, "Resilient optical network technologies for catastrophic disasters," Journal of Optical Communications and Networking, vol. 9, no. 6, pp. A280-A289, 2017.

[40] A. Agrawal, V. Bhatia, and S. Prakash, "Network and risk modeling for disaster survivability analysis of backbone optical communication networks," Journal of Lightwave Technology, vol. 37, no. 10, pp. 2352 $2362,2019$.

[41] A. Kwasinski, "Effects of notable natural disasters from 2005 to 2011 on telecommunications infrastructure: Lessons from on-site damage assessments," in Int. Telecommunications Energy Conference (INTELEC). IEEE, 2011, pp. 1-9.

[42] J. Hershberger and S. Suri, "An optimal algorithm for euclidean shortest paths in the plane," SIAM Journal on Computing, vol. 28, no. 6, pp. 2215-2256, 1999.

[43] J. S. Mitchell et al., "Geometric shortest paths and network optimization," Handbook of computational geometry, vol. 334, pp. 633-702, 2000.

[44] D. Z. Chen and H. Wang, "Computing shortest paths among curved obstacles in the plane," ACM Transactions on Algorithms (TALG), vol. 11 , no. 4 , p. $26,2015$.

[45] I.-K. Lee, M.-S. Kim, and G. Elber, "Planar curve offset based on circle approximation," Computer-Aided Design, vol. 28, no. 8, pp. 617-630, 1996.

[46] H. Persson, "Nc machining of arbitrarily shaped pockets," ComputerAided Design, vol. 10, no. 3, pp. 169-174, 1978.

[47] R. Farias and M. Kallmann, "Optimal path maps on the gpu," IEEE Transactions on Visualization and Computer Graphics, 2019.

[48] G. Jameson, "Inequalities for the perimeter of an ellipse," The Mathematical Gazette, vol. 98, no. 542, pp. 227-234, 2014.

[49] P. K. Agarwal, A. Efrat, S. K. Ganjugunte, D. Hay, S. Sankararaman, and G. Zussman, "The resilience of wdm networks to probabilistic geographical failures," IEEE/ACM Transactions on Networking (TON), vol. 21, no. 5, pp. 1525-1538, 2013.

[50] S. Knight, H. Nguyen, N. Falkner, R. Bowden, and M. Roughan, "The internet topology zoo," Selected Areas in Communications, IEEE Journal on, vol. 29, no. 9, pp. $1765-1775$, october 2011.

[51] S. Orlowski, M. Pióro, A. Tomaszewski, and R. Wessäly, "SNDlib 1.0Survivable Network Design Library," in Proc. INOC, April 2007.

[52] "Telia carrier map USA." [Online]. Available: https://www.teliacarrier. com/our-network.html

[53] "Tata communications, India \& neighbouring countries network." [Online]. Available: https://www.tatacommunications.com/map/ 